\title{
Promoting Fall Prevention among Community Dwelling Older Adults through ActivLife: a Physical and Social Activation
}

\author{
Yuan Lu ${ }^{1}$ (D) Yu-Shan Athena Chen ${ }^{1}$ (D) Dominika Kozak ${ }^{2}$ (D) \\ Hubert Cornelis $^{1,3}$ (D) Piotr Partyga ${ }^{2}$
}

Received: 29 October 2019 / Accepted: 15 January 2020 / Published online: 12 February 2020

(C) The Author(s) 2020

\begin{abstract}
Falls have been recognized as the second leading cause of injury or death for older adults. The related economic burden caused by fall related injuries is not negligible. Earlier research has demonstrated that regular participation in appropriate prescribed physical activity by improving upper and lower limb strength, balance, coordination, transfer skills, and reaction to environmental hazards can lower the risk for falls and fall-related fractures and other injuries. Conversely, physical inactivity can significantly double the risk of developing a disability, which will affect mobility as well as the ability to perform even the most basic activities of daily life, therefore, ultimately increases the older adults' risk for falls.

This paper first presents a technological solution ActivLife that aims at preventing older adults from falling through practicing physical training in a safe and playful manner, followed by a randomized controlled study with 43 older adults with an average age of 77 for a period of 16 weeks in a social activation center het Ontmoet en Groethuys in Eindhoven, the Netherlands to demonstrate to what extent ActivLife could help to prevent falling among older adults.
\end{abstract}

Keywords Fall prevention · Social support · Community-dwelling older adults $\cdot$ Physical activities

Yuan $\mathrm{Lu}$

y.lu@tue.nl

1 Department of Industrial Design, Eindhoven University of Technology, Noord Brabant, Eindhoven, Netherlands

2 Arleh Medical, Lodz, Poland

3 Ontmoet en Groethuys, Eindhoven, the Netherlands 


\section{Introduction}

According to United Nations, the population percentage of $60+$ older people in Europe would rise from more than $20 \%$ in 2017 to almost $35 \%$ in 2050 due to the increasing life expectancies and decreasing fertility rates (United Nations - Population Division Department of Economic and Social Affairs 2017). Consequently, the ratio between the number of older people, aged 65 and above, and the number of working-age citizens will also increase (Eurostat 2017) and result in an enormous amount of pressure on EU's healthcare systems funded mainly by the working-aged generation, i.e., the taxpayers.

The health expenditure in the EU will rise to $350 \%$ by 2050 , according to the Organization for Economic Cooperation and Development (Espinoza 2011), primarily contributed by the expenditure relates to the group of older adults (Eurostat 2016). This increase is because that older people are more likely to suffer from multiple comorbid conditions and chronic diseases and therefore require more curative, rehabilitation, and long-term care (LeRouge et al. 2013; Sneha and Varshney 2009).

According to the World Health Organization, falls have been recognized as the second leading cause of accidental or unintentional injured death globally. Adults older than 65 years suffer from the most significant number of fatal or non-fatal falls (World Health Organisation 2018). Falls occur as a result of a complex combination and interaction of several risk factors, including biological, behavioral, environmental, socioeconomic factors (World Health Organisation 2007):

-Biological factors associated with several non-modifiable biological factors relating to falls, such as age, gender, and race, as well as the aged related declines in physical, cognitive, and affective conditions such as postural stability in combination with impaired mobility, gait, and balance (Morrison 2013).

-Behavioral risk factors relate to human actions, emotions, and daily choices that lead to falls, therefore, potentially modifiable.

-Environment risk factors lie in hazardous situations at home and the public environment.

-Socioeconomic risk factors refer to those social conditions and economic of individuals and the capacity of the community.

"Indoor falls" are given more research attention than to falls occuring outdoor. Those who fall outdoors are considered to be healthier and more fit than those who fall indoors (Satariano et al. 2017). The typical faller profile described in current literature is frail older adults who commonly fall indoors and healthy older adults who fall outdoors mostly.

EuroSafe and ProFouND reported that each year in the EU, there are about 36,000 older people reported to get fatally injured from falls, 1,443,000 fall-related injuries admitted to hospital, and 2,314,000 older people attending emergency departments with fall-related injuries (EuroSafe and ProFouND 2015). Besides, falling among older adults may also result in a psychological trauma called fear of falling. Such fear may lead to self-restriction of activities and staying at home, which harms the physical condition of the elderly (Bruce et al. 2002; Li et al. 2003; Tinetti et al. 1988; Zijlstra et al. 2005). It is known that fear of falling is often associated with the prior experience of fall (Arfken et al. 1994). Fear of falling is, therefore, a health problem of older adults and can be observed even in older adults who never fall. Fear of falling may cause a 
decrease in functional activities and is strongly connected with adverse outcomes such as functional decline, falls, and depression. Activity limitation due to fear of falling may cause loss of independence and reduce the social interaction, which in turn leads to physical inactivity and lower quality of life (Dias et al. 2011). Older adults whose activities are limited by fear of falling are at a higher risk of falls because activity restriction may lead to functional decline and increased risk of falls in the future. Dias et al. (Dias et al. 2011) emphasized that variables such as depression, exhaustion, and social activity connected strongly with activity limitation due to sadness and fear of falling. Reducing fear of falling can contribute to prevent and limit the consequences of falls among older adults (Arfken et al. 1994).

Regular physical activity is associated with better mobility, improved daily activities, as well as decreased anxiety and occurrence of depression in people over the age of 65 (Evans 1999; Leveille et al. 1999; Psaltopoulou et al. 2008). In particular, physical exercise can play an important role in fall prevention and reduce the number of injurious falls (Guirguis-Blake et al. 2018; Jorgensen et al. 2013; Pereira et al. 2008). Unfortunately, the daily activities of older adults are not yet sufficient to meet the World Health Organization recommendations on the required minimum fitness levels (Halat et al. 2014).

Hamm et al. (2016) provided an overview of falls prevention interventions for community-dwelling older people, including supervised and unsupervised exercise, education interventions, fall risk assessment, home assessment/assistive equipment, and technology-based interventions (Hamm et al. 2016). Many issues exist related to the adherence and acceptance of the range of both supervised and unsupervised exercise interventions. Supervised exercises require a lot of time and costs from both the exercise providing and receiving side. The support received in supervised activities, however, can help reduce the fear of falling. Unsupervised home-based training relies very much on the individual's intrinsic motivations and disciplines and is lack of interaction with others and personalization (Geraedts et al. 2014). Yet the fear of falling is not necessarily addressed in unsupervised home-based training.

Evans (1999) found that the use of simple forms of movement in such a way to associate physical activities with pleasure, relaxation, and fun is most effective in stimulating physical activities among seniors. Specialized intervention programs are needed to properly stimulate physical activities related to fall prevention, such as muscle strength or dynamic balance, as well as cognitive factors (Leś et al. 2017). Therefore, many interventions have been developed to stimulating physical exercises and reducing the fear of falling (Gillespie et al. 2012; Patel et al. 2012; Sherrington et al. 2008; World Health Organisation 2007; Zijlstra et al. 2005). Furthermore, increasingly more technology-assisted solutions are available to identify and analyze falling risks, detect falls, and inform caregivers. Earlier work by (Abbate et al. 2012; Cao et al. 2012; Garcia et al. 2014; Silva et al. 2013; Yu et al. 2012) used the measurement of movement and postures based on (wearable) sensor and smartphone technology to enrich the exercise experiences. More tailored and interactive exercise programs based on 3D technology and games have been used to improve exercise adherence as well (Shubert 2011; Taylor et al. 2012; Uzor et al. 2013; Williams et al. 2010).

The ActivLife device is a technology-assisted solution to activate the physical and mental activity of older adults. ActivLife can be used to prevent falls and provide an 
adequate support for cognitive processes due to the simultaneous combination of the physical and mental exercises. With a mechanism assisting in standing up called GymUp, ActivLife supports mobility exercises of the ankle, knee, and hip joints. It aims at increasing muscle strength of the lower limbs while performing squats and back stances. With a unique corset and seat, it ensures safety during exercises when older adults strengthen the back and abdomen muscles by lifting the legs. ActivLife also supports to maintain a safe, upright standing position when performing balancing exercises as well as exercises activating the upper parts of the body using a gamebased multimedia program (Fig. 1). The 3-D technology and game-based interactive multimedia program aim at effectively supporting cognitive processes during physical and mental exercises (Fig. 2).

This paper is interested in finding out the potential of applying ActivLife device as a technology-assisted exercise intervention solution in the supervised condition to motivate physical exercises and prevent risks of falls for community-dwelling older adults. The paper is organizialed as follows. A detailed discussion of the research method of setting up the randomized controlled trial, the hypotheses, the related measurement, and analysis methods is given first. The experiment results and analysis are reported after that. Discussion and conclusion are given in the end.

\section{Research Method}

\section{Sample Size, Recruitment and Experiment Set up}

We conducted a randomized controlled trial. A senior community center, Ontmoet en GroetHuys in Eindhoven, which we worked with sent out a news letter in which they called for participation. The interested older adults could register for this experiment at the community center. These old adults all lived in their own home close to the community center. Ideally, large sample size should be chosen to ensure statistical significance of the experiment results. In practice, the number of participants in this study was restricted because of participant availabity, cost and time considerations.

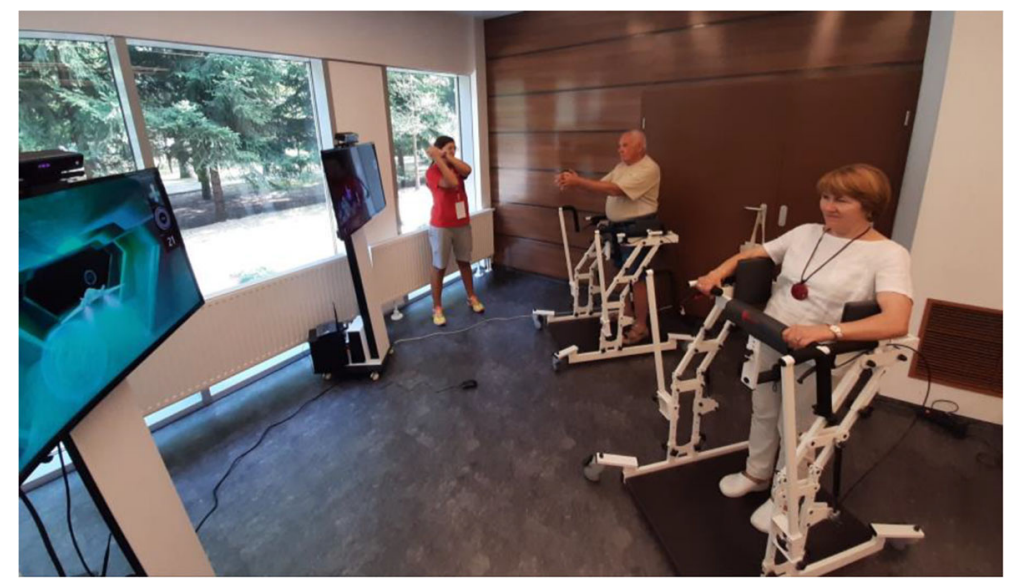

Fig. 1 Older adults use ActivLife in a safe and upright stand position 


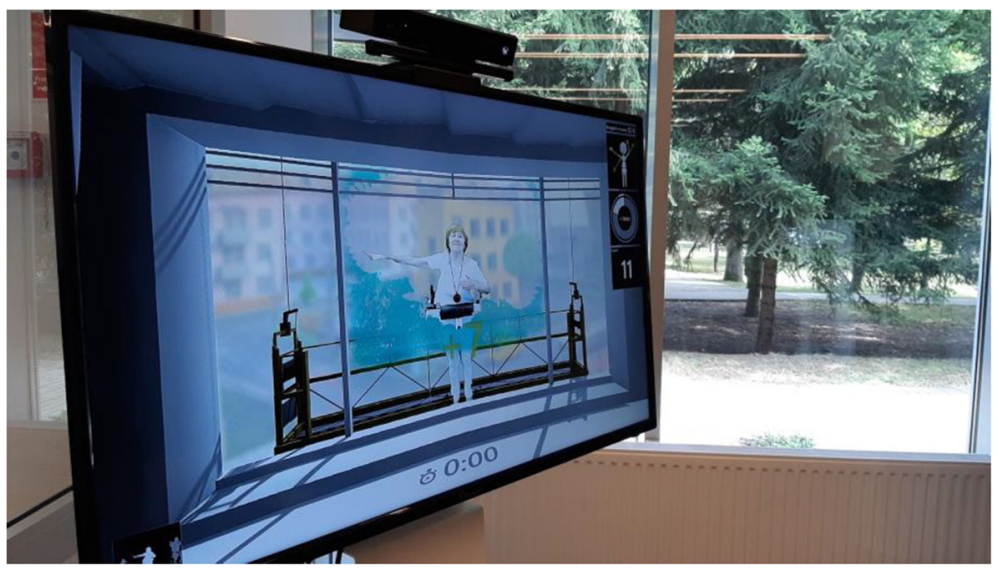

Fig. 2 ActivLife uses 3-D technology and a game-based multimedia platform

There were about 150 older adults visiting the community center. Therefore, it was not feasible to start to recruit the large sample normally required for such an experiment.

In order to make fall risks comparable and prevent unnecessary injury, we would only include participants who were not frail. After the selection, we would randomly assign the participants in two groups. Block randomization technique was used. The participants would be assigned to a number of blocks of 2 participants per block. We randomized these blocks and made sure that each time 1 participant would be assigned to group A and the other participant would be assigned to group B. If the total number of participants are not able to be divided by 2, the last participant would be just assigned to either of the groups. Group A would involve in training with the ActivLife device for eight weeks. They would train twice a week for 30 min each time supported by a sport coach. They would receive a test map consisting of the following items.

- Introduction and explanation of the exercises.

- Training program for neck-shoulder-back and a personalized time program for each participant with the support of the sport coach.

Group B would conduct a home exercise based on the written instruction and advice from the physiotherapist for eight weeks. They would receive a test map which consisted of the following information.

- Introduction and explanation of the exercises.

- Exercises at home to improve mobility of the neck-shoulder-back.

For both groups, the exercise they received from either ActivLife or physiotherapist would remain the same during the test period.

\section{Measurement}

Due to rapidly aging, frailty has become an essential concept for human-being, as it identifies older people who have a higher risk of adverse outcomes, such as disability, 
institutionalization, lower quality of life, and premature death. The Tilburg Frailty Indicator (TFI) (Gobbens et al. 2010) is based on a multidimensional approach to frailty. It measures frailty by assessing physical, psychologic, and social aspects of human functioning. In order to check whether the participants have a similar frailty level, the frailty level of each participant was measured using TFI before the program. When TFI is larger than 5, the older adult is considered frail. We used TFI as an inclusion criteria to recruit participations and therefore only measure it once before the test.

We used Stage of change questionnaire (SCQ) based on Transtheoretical Model (DiClemente and Prochaska 1998; Prochaska and Velicer 1997) before and after the interventions to measure the change in behavior due to the interventions. This SCQ helps the older adults to allocate themselves along the behavior change continuum towards being more physically active: pre-contemplation, contemplation, preparation, action, and maintenance. In the pre-contemplation stage, the older adults may not consider change at all and may not see inactive as a problem for them. The contemplation stage reflects that the older adults are becoming open to the idea of change but might feel ambivalent about it. They may frequently change their minds about whether or not to be active is a "good" idea. Once in the preparation stage, older adults have committed to change and begin to consider lifestyle changes that need to be made. It is not uncommon in this stage for them to seek out support from therapists or other sources. While older people in the maintenance stage, they are focusing on maintaining changes that have been made.

Martins et al. (2018) proposed to use a multifactorial, instrumented, screening tool for assessing fall risks based on several functional tests. Given the time and resources, we chose to conduct Hand Grip Test (HGT) (Desrosiers et al. 1995; Pijnappels et al. 2008), the 30 Seconds Sit to Stand (30STS) test (Shirly Ryan Ability Lab 2013), 4-stage of balance test (Martins et al. 2018) and the Tinetti Balance Assessment (TBA) (Tinetti et al. 1988) in our experiment which are important for fall assessment and identification.

During the 30STS test, the person crosses his arms on his/her chest, then stands and sits repetitively for $30 \mathrm{~s}$. Therefore, the 30STS Test assesses lower extremity strength and endurance. The test score is the number of times that the person comes to a full standing position in $30 \mathrm{~s}$. (Centers for Disease Control and Prevention and Injuries 2017) provides a table of a below average scores that indicates the risk for falls for different older age groups.

The 4-Stage of Balance Test assesses static balance in four different and increasingly challenging positions. They are feet together, instep of foot advanced to toe of other foot, foot in front of other foot (tandem), and single leg stance. Success is maintenance of each position for $10 \mathrm{~s}$; less than $10 \mathrm{~s}$ indicates stage failure. Passing is completion of the third stage for 10 or more seconds. The TBA is a simple but the olderest clinical balance test to measure the gait and used widely among older adults (Anon n.d.-a). It results in a total score based on assessments of both gait and balance. When the total score is higher and equal to 24 , the risk of falling is low. When the total score is between 19 and 23, there is a moderate risk of falling present. When the total score is smaller and equal to 18 , there is a high risk of falling present. We conducted these tests before and after the intervention for each group to be able to identify possible functional improvement, which could potentially contribute to falling prevention as well as to compare the intervention effect across the groups.

We also measured the Barriers to Being Active (BBA) before and after the intervention to understand how the interventions could influence the BBA and whether 
group A has lower barriers to be active after using ActivLife compared to group B after an unsupervised training program provided by physiotherapists at home. We applied the questionnaire with 21 items developed by the Centers for Disease Control and Prevention (Centers for Disease Control and Prevention n.d.) in which the following categories of barriers were measured (Sawchuk et al. 2011).

- Lack of time: there is insufficient time to be active.

- Lack of social influence: there is a need for social influence to be active.

- Lack of energy: there is lack of energy to be active.

- Lack of motivation: there is lack of motivation to be active.

- Fear of injury: Fear of injury is the barrier to be active.

- Lack of skill: there is a need for skill to be active.

- Lack of resources: there is a need for resources to be active.

When the BBA score in any category is equal or higher than 5, it means that this is an important barrier to overcome. Table 1 below summarizes the measurements made during the experiment.

\section{Hypotheses}

We introduce formal hypotheses regarding the role of ActivLife device in promoting the physical activities of older adults.

First, we propose that the interventions have no influence on the Stage of Change for both groups.

H1: Group A remains in the same stage of change before and after the intervention.

$\mathrm{H} 2$ : Group B remains in the same stage of change before and after the intervention.

Concerning physical strength measured by HGT, the 30-s STS, and the 4-Stage Balance Test and TBA, we propose that both groups retain their physical strength before and after both interventions. The hypotheses are as follows.

H3: The HGT results remain unchanged for Group A before and after the intervention. H4: The HGT results remain unchanged for Group B before and after the intervention.

Table 1 Measurements during the randomized controlled trial for both groups

\begin{tabular}{lll}
\hline & Baseline & \\
\hline Age, gender & $\mathrm{X}$ & Intervention \\
TFI & $\mathrm{X}$ & \\
HGT & $\mathrm{X}$ & $\mathrm{X}$ \\
SCQ & $\mathrm{X}$ & $\mathrm{X}$ \\
30-s STS & $\mathrm{X}$ & $\mathrm{X}$ \\
TBA & $\mathrm{X}$ & $\mathrm{X}$ \\
BBA & $\mathrm{X}$ & $\mathrm{X}$ \\
\hline
\end{tabular}


H5: The 30-s STS results remain unchanged for Group A before and after the intervention.

H6: The 30-s STS results remain unchanged for Group B before and after the intervention.

H7: The TBA results remain unchanged for Group A before and after the intervention. H8: The TBA results remain unchanged for Group B before and after the intervention.

Finally, we propose that Group A has an equal or higher Barrier to be Active compared to Group B after the intervention. Thus, the corresponding hypotheses are:

H9: Group A scores an equal score on lack of time compared to Group B after the intervention.

H10: Group A scores an equal score on lack of social influence compared to Group B after the intervention.

H11: Group A scores an equal score on lack of energy compared to Group B after the intervention.

H12: Group A scores equal score on lack of motivation compared to Group B after the intervention.

H13: Group A scores an equal score on fear of injury compared to Group B after the intervention.

H14: Group A scores an equal score on lack of skills compared to Group B after the intervention.

H15: Group A scores an equal score on lack of resources compared to Group B after the intervention.

\section{Ethical Consideration}

All participants were asked to sign a consent form with the research team, and the client board of the community center has approved the test protocol. All data collected was saved in two steps. The participants' information was stored offline at the community center while each participant was assigned a specific participant number. The collected measurements per participant were saved anonymized in an excel file while a metafile was created to explain how to read the data from this excel file. This data would be only used in the project context as agreed with the participants.

\section{Results}

\section{Participants}

In total, eighty older adults reacted to the call for participation. Among them, thirtyseven participants had a TFI score higher than five. They were considered as frail, therefore excluded from the experiement. Only forty-three participants (nineteen females and twenty-four males), with an average age of 76.9 and a standard deviation of 7.5 , participated in the experiment. The participants were assigned randomly to two intervention conditions. Group A consisted of eleven females and ten males, with an 
Table 2 Participants information

\begin{tabular}{llll}
\hline & Average age & Number of female participants & Number of male participants \\
\hline Group A & 78.1 & 11 & 10 \\
Group B & 75.8 & 8 & 14 \\
\hline
\end{tabular}

average age of 78.1. Group B consisted of 8 females and 14 males, with an average age of 75.8. Table 2 summarizes the participants' information.

\section{Measures}

The hypotheses were examined by bootstrapping method (2000 boostratp sample), which allows estimation of the sampling distribution, especially for small samples. The effect sizes (Cohen's $d$ ) were reported. Cohen (1988) describes an effect size of 0.2 as 'small,' 0.5 as "medium," and 0.8 as "large."

The TFI scores were measured at the beginning of the experiment for both groups. The results showed that the average TFI of group $\mathrm{A}=3.47$ and the average TFI of group $\mathrm{B}=3.22$. Since the TFIs of two groups are not normally distributed, we performed an Independent-Samples Mann-Whitney U Test with $p=.95$ and, therefore, cannot reject the fact that the distribution of TFI is the same across the two groups with $95 \%$ confidence. This result implies that these two groups have a statistical comparable frailty level before the experiment.

\section{SCQ}

Participants in group A were initially between the stage of decision and the stage of action. We conducted a paired-samples t-test and learned that they remained at the same stage after the intervention ( $p=1>.05$, Cohen's $d=.00)$, their motivation to move has remained. Participants in group B were initially between the stage of action and the stage of maintenance. We conducted a paired-samples t-test. We learned that they evaluated themselves to be between the stage of decision and stage of action after the intervention ( $p=.06<.10$, Cohen's $d=.45)$, i.e., their motivation to move has reduced.

\section{HGT}

We conducted mixed-design ANOVAs with two interventions as a between-subject variable to examine whether the interventions influenced right- and left-hand grip strength (HGS) (comparing the pre- and post-results). The results demonstrated that none of the HGS was statistically significantly changed. With regard to Group A, ActivLife exercise did not influence the right-HGS ( $p=.10$, Cohen's $d=.44$ ) or leftHGS ( $p=.59$, Cohen's $d=.13$ ). Similar results were found among Group B (rightHGS: $p=.55$, Cohen's $d=-.14$; left-HGS: $p=.66$, Cohen's $d=.10$ ). 


\section{0-S STS}

We conducted two paired-samples t-tests on 30-s STS among Group A and B. The results indicated that ActivLife exercises enhanced Group A participants' 30-s STS (pre-test mean $=11.20$; post-test mean $=13.33, t=2.27, p<.05$, Cohen's $d=.59$ ). Since the average ages of the test participants are between 75 and 79 and the STS scores of both before and after the test were higher than 11, these participants have no indicator of risk for falls (Centers for Disease Control and Prevention and Injuries 2017). Thus, H5 could be rejected with $95 \%$ confidence. Regarding Group B, the findings demicipantsonstrated that the 30-s STS performances were also improved among the participants who had unsupervised hometraining (pre-test mean $=11.20$; post-test mean $=13.30, t=2.27, p<.05$, Cohen's $d=.49$ ). Since the average ages of the test participants are between 75 and 79 and the STS scores of both before and after the test were higher than 11, these participants have no indicator of risk for falls (Centers for Disease Control and Prevention and Injuries 2017). Therefore, H6 could be rejected with $95 \%$ confidence.

\section{4-Stage of Balance Test}

We conducted two paired-samples t-tests on the 4-stage of balance test among Group A and Group B. The results indicated that ActivLife exercise did not improve the 4-stage of balance measurement significantly (pre-test mean $=34.56$, post-test mean $=36.11$, $t=-1.25, p=0.2>0.05$, Cohen's $d=.29$ ) and neither the unsupervised home-based training (pre-test mean $=38.05$, post-test mean $=38.15, t=-0.124, p=0.90>0.05$, Cohen's $d=.03$ ). Therefore, $\mathrm{H} 7$ could not be rejected with $95 \%$ confidence, either.

\section{TBA}

The summary of the assessment results of two groups is listed in the table below. We can conclude that all participants are not at a high risk of falling when the experiment was conducted (Table 3).

We conducted paired-sample t-tests to compare the TBA score on the total balance level before and after the intervention. We learned that 1) supervised exercises did improve the total balance level with $(p=0.039<0.05$, Cohen's $d=.53) ; 2$ ) Unsupervised home-training did improve the total balance level $(p=0.005<0.05$, Cohen's $d=.71$ ). Therefore we could reject $\mathrm{H} 7$ and $\mathrm{H} 8$ with $95 \%$ confidence.

Table 3 TBA results

\begin{tabular}{llll}
\hline & & Mean & Standard deviation \\
\hline \multirow{2}{*}{ Group A } & Before & 24.6 & 3.90 \\
& After & 25.9 & 3.80 \\
\multirow{2}{*}{ Group B } & Before & 26.0 & 2.30 \\
& After & 27.2 & 2.13 \\
\hline
\end{tabular}




\section{BBA}

Lack of Time H9 regards whether participants in Group A complained less about lack of time than the ones in Group B after the interventions. Thus we conducted an independent t-test and learned that participants in Group A did not report less lack of time than Group B after the intervention. We did not have significant results to reject H9 $(t=.90, p=.37$, Cohen's $d=.30)$ with $95 \%$ confidence.

Lack of Social Influence H10 considers whether Group A complained less about the lack of social influence than Group B after interventions. We conducted an independent t-test and learned that participants in Group A reported less about the lack of social influence than Group B after the intervention. With this result, we could reject H9 $(t=1.92, p=.06$, Cohen's $d=.64)$ with $90 \%$ confidence.

Lack of Energy H11 suggests whether Group A complained less about the lack of energy than Group B after interventions. An independent t-test was conducted. From the results, we learned that participants in Group A did not complain less about the lack of energy than Group B after the intervention. With this result, we could not reject H11 $(t=1.32, p=.19$, Cohen's $d=.44)$ with $95 \%$ confidence.

Lack of Motivation H12 aims to examine whether Group A complained less about the lack of motivation than Group B after interventions. An independent t-test was conducted. The results demonstrated that participants in Group A did not complain less about the lack of motivation than Group B after the intervention. With this result, we could not reject H12 ( $t=.03, p=.98$, Cohen's $d=.01)$ with $95 \%$ confidence.

Fear of Injury H13 aims to examine whether Group A complained less fear of injury than Group B after interventions. An independent t-test was conducted. The results demonstrated that participants in Group A reported did not complain less fear of injury than Group B after the intervention. With this result, we could not reject H13 $(t=.02$, $p=.98$, Cohen's $d=.01$ ) with $95 \%$ confidence.

Lack of Skill H14 aims to examine whether Group A less complained about the lack of skill than Group B after interventions. An independent t-test was conducted. The results demonstrated that participants in Group A did not complain less about their lack of skill than Group B after the intervention. With this result, we could not reject H14 ( $t=1.23$, $p=.22$, Cohen's $d=.41$ ) with $90 \%$ confidence.

Lack of Resources H15 aims to examine whether Group A less complained about the lack of resources than Group B after interventions. An independent t-test was conducted. The results demonstrated that participants in Group A did not complain less about their lack of resources than Group B after the intervention. With this result, we could not reject H15 $(t=1.09, p=.28$, Cohen's $d=.37)$ with $90 \%$ confidence. 


\section{Discussion and Conclusion}

We learned that following the supervised ActivLife exercise did not lead to the improvement of HGS. Future activities such as the five exercises to improve hand strength recommended by Dobrosielski (Anon n.d.-bcan be designed in order to improve HGS as a part of the fall prevention program, which is currently missing in the training program.

ActivLife exercise has an apparently significant impact on improving the 30 STS and TBA. It implies that ActivLife focuses very much on training the core and lower body strength, which is of great importance to fall prevention. However, neither the supervised ActivLife exercise or the unsupervised home-based training bemonstrated any improvement of the 4-stage of balance test. The participants had quite good 4-stage balance scores before the test, which is an indication of a moderate to low level of risk of falling. With the biased sample, it was not feasible to conclude on the effect of ActivLife on improving 4-stage of balance test. In addition, although ActivLife seems to improve the TBA largely, from the TAB results in Table 2, we learned that our participants had a moderate to low level of risk of falling. Therefore, we could not conclude that ActivLife could indeed improve the TAB because of the biased sample. Further research is needed to examine ActivLife with groups at high risk of falling.

From the reported SCQ results, we learned that the participants felt that the supervised ActivLife exercise had motivated them more towards physical activities, and they were ready to take action to be and remain active. The participants of the unsupervised home-training program felt less motivated. Apparently, the sports coach had a very important role in motivating the participants to remain active and be more active. This result suggested that social support was an essential stimulus for these participants to perform their daily activities. We confirmed this finding with the results of the BBA analysis between these two groups: supervised ActivLife exercise offered the social support that the older adults were in need when exercising, but the unsupervised home training program did not.

Yet, we have to mention that all SCQ results showed that both groups were in the stage of making decisions to change, taking actions or working on maintenance. Besides, the BBA scores of both groups are significantly smaller than 5, i.e., they do not experience any related barriers measured with $95 \%$ statistical significance (all $p=$ $0.00<0.05$ ). This result implies that we have recruited a somewhat motivated and active group for this experiment. It is, therefore, no surprise to see that TBA was pretty much improved under both interventions. It would be interesting to perform the test with a group that is less active and less motivated.

In sum, the most important conclusion we can draw from this study is that a capable technological training program can only be successful for community-dwelling older adults if it is offered as an intervention in a social set up. The resulted social interactions can serve as intervention trigger points and enable them to show others "best practices," which can be a model to follow by older adults who start to be active. Last but not least, the number of participants in the experiment was really small to generalize the obtained results beyond the context. The test period was also rather short to really see the effects of the interventions. Future research with ActivLife should, therefore, focus on involving more participants for a longer period of time, e.g. up to 3 months, to possibly see differences. 
Acknowledgements We, the authors of this work, would like to thank the REACH2020 project as well as our partners at the senior community center. The research leading to these results presented in this article has received funding from the European Union's Horizon 2020 research and innovation programme under grant agreement No 690425. Besides, we would like to extend our sincerest gratitude to the men and women who shaped our contribution possible; the participants, the volunteers of the senior community center, and the physiotherapists from MaxFysio Waarlre.

Open Access This article is licensed under a Creative Commons Attribution 4.0 International License, which permits use, sharing, adaptation, distribution and reproduction in any medium or format, as long as you give appropriate credit to the original author(s) and the source, provide a link to the Creative Commons licence, and indicate if changes were made. The images or other third party material in this article are included in the article's Creative Commons licence, unless indicated otherwise in a credit line to the material. If material is not included in the article's Creative Commons licence and your intended use is not permitted by statutory regulation or exceeds the permitted use, you will need to obtain permission directly from the copyright holder. To view a copy of this licence, visit http://creativecommons.org/licenses/by/4.0/.

\section{References}

Abbate S, Avvenuti M, Bonatesta F, Cola G, Corsini P, \& Vecchio A (2012). A smartphone-based fall detection system. Pervasive and Mobile Computing, 8(6), 883-899.

Anon (n.d.-a) "5 Exercises That Increase Hand Grip Strength - IBX Events." Retrieved November 21, 2019 (https://events.ibx.com/5-exercises-increase-hand-grip-strength/).

Anon (n.d.-b) “Tinetti Balance Assessment Tool.” Retrieved October 22, 2019 (http://hdcs.fullerton. edu/csa/Research/documents/TinettiPOMA.pdf).

Arfken CL, Lach HW, Birge SJ, \& Miller JP (1994). "The prevalence and correlates of fear of falling in elderly persons living in the community." American Journal of Public Health, 84(4), 565-570.

Bruce DG, Devine A, \& Prince RL (2002). "Recreational physical activity levels in healthy older women: The importance of fear of falling." Journal of the American Geriatrics Society, 50(1), 84-89.

Cao, Y, Yang Y, \& Liu W H. (2012). "E-FallD: A Fall Detection System Using Android-Based Smartphone." Proceedings of 2012 9th International Conference on Fuzzy Systems and Knowledge Discovery, FSKD 2012, 1509-1513.

Centers for Disease Control and Prevention (n.d.) "Barriers to Being Active Quiz What Keeps You from Being More Active?" https://www.cdc.gov/physicalactivity/basics/adding-pa/barriers.html. Accessed 21 Sep 2019.

Centers for Disease Control and Prevention and Stopping elderly accidents Deaths \&. Injuries (2017). “ASSESSMENT 30-Second Chair Stand Patient.” Retrieved November 21, 2019 (https://www.cdc. gov/steadi/pdf/STEADI-Assessment-30Sec-508.pdf).

Cohen, J. (1988). Statistical power analysis for the behavioral sciences. Routledge.

Desrosiers J, Bravo G, Hébert R, \& Dutil E (1995). "Normative data for grip strength of elderly men and women." The American Journal of Occupational Therapy: Official Publication of the American Occupational Therapy Association, 49(7), 637-644.

Dias RC, Freire MTF, Santos ÉGS, Vieira RA, Dias JMD, \& Perracini MR (2011). "Characteristics associated with activity restriction induced by fear of falling in community-dwelling elderly." Brazilian Journal of Physical Therapy, 15(5), 406-413.

DiClemente CC, and Prochaska JO (1998). "Toward a comprehensive, transtheoretical model of change." In: pp. 3-24, Treating Addictive Behaviors. Springer US.

Espinoza, J (2011). "Europe's failing health." The Wall Street Journal. https:/www.wsj. com/articles/SB10001424052748704893604576200724221948728. Accessed 21 Sept 2019.

EuroSafe and Pro FouND (2015). Falls among Older Adults in the EU-28: Key Facts from the Available Statistics.

Eurostat (2016). "Current healthcare expenditure relative to GDP, 2016." Statistics Explained. Retrieved October 2, 2019 (https://ec.europa.eu/eurostat/statistics-explained/index.php/Healthcare_expenditure_ statistics\#Health_care_expenditure). 
Eurostat (2017). "Population structure and ageing." Statistics Explained. Retrieved September 23, 2019 (https://ec.europa.eu/eurostat/statistics-explained/index.php/Population_structure_and_ageing\#Slightly_ more_than_three_persons_of_working_age_for_every_person_aged_65_or_over).

Evans, William J (1999). "Exercise Training Guidelines for the Elderly." Medicine and Science in Sports and Exercise, 31, 12-17.

Garcia JA, Pisan Y, Tan CT, Navarro KF (2014). “Assessing the Kinect's Capabilities to Perform a TimeBased Clinical Test for Fall Risk Assessment in Older People.” In: Pisan Y., Sgouros N.M., Marsh T. (eds) Entertainment Computing - ICEC 2014. ICEC 2014. Lecture Notes in Computer Science, vol 8770, 100107. Springer, Berlin, Heidelberg

Geraedts HA, Zijlstra W, Zhang W, Bulstra S, \& Stevens M (2014). "Adherence to and effectiveness of an individually tailored home-based exercise program for frail older adults, driven by mobility monitoring: Design of a Prospective Cohort Study.” BMC Public Health, 14, 570.

Gillespie LD, Clare Robertson M, Gillespie WJ, Sherrington C, Gates S, Clemson LM, \& Lamb SE (2012). "Interventions for preventing falls in older people living in the community. The Cochrane database of systematic review." The Cochrane Database of Systematic Reviews, 9, CD007146.

Gobbens RJJ, van Assen MALM, Luijkx KG, Wijnen-Sponselee, M. T., \& Schols, J. M. G. A. (2010). "The Tilburg frailty Indicator: Psychometric properties." Journal of the American Medical Directors Association, 11(5), 344-355.

Guirguis-Blake, J. M., Michael, Y. L., Perdue, L. A., Coppola, E. L., Beil, T. L., Thompson, J. H. (2018). Interventions to prevent falls in community-dwelling older adults: A systematic review for the U.S. Preventive Services Task Force. Rockville (MD): Agency for Healthcare Research and Quality (US), (Evidence Synthesis, No. 159.) https://europepmc.org/books/NBK525700;jsessionid=0A5DA113E2B2 E051406EE818629C8762. Accessed 21 Dec 2019

Halat B, Brudz D, Milewicz K, Pop T, \& Śliwiński Z (2014). "The impact of general fitness exercises on balance and gait of elderly people staying in the ZOL branch in Legnica.” Medical Review, 1, 84-96.

Hamm J, Money AG, Atwal A, \& Paraskevopoulos I (2016). "Fall prevention intervention technologies: A conceptual framework and survey of the state of the art." Journal of Biomedical Informatics, 59, 319-345.

Jorgensen MG, Laessoe U, Hendriksen C, Nielsen OBF, \& Aagaard P (2013). "Efficacy of Nintendo Wii training on mechanical leg muscle function and postural balance in community-dwelling older adults: A randomized controlled trial." Journals of Gerontology - Series A Biological Sciences and Medical Sciences, 68(7), 845-852.

LeRouge C, Ma J, Sneha S, \& Tolle K (2013). "User profiles and personas in the design and development of consumer health technologies." International Journal of Medical Informatics, 82(11), e251-e268.

Leś A, Niedzielska E, Piotrowska J, Staniszewski M, \& Kozak D (2017). "Analysis of the effectiveness of ActivLife training in people over 60 years of age: A pilot study." Gerontechnology, 16(3), 189-195.

Leveille SG, Guralnik JM, Ferrucci L, \& Langlois JA (1999). "Aging successfully until death in old age: Opportunities for increasing active life expectancy.” American Journal of Epidemiology, 149(7), 654 664.

Li F, John Fisher K, Harmer P, McAuley E, \& Wilson NL (2003). "Fear of falling in elderly persons: Association with falls, functional ability, and quality of life." Journals of Gerontology - Series B Psychological Sciences and Social Sciences, 58(5), 283-290.

Martins AC, Moreira J, Silva C, Silva J, Tonelo C, Baltazar D, Rocha C, Pereira T, \& Sousa I (2018). "Multifactorial screening tool for determining fall risk in community-dwelling adults aged 50 years or over (FallSensing): Protocol for a prospective study." Journal of Medical Internet Research, 7(8): e10304.

Morrison S (2013). "Falling down: Assessing the risk of falls in older adults." Age in Action, 28(3), 1-6.

Patel S, Park H, Bonato P, Chan L, \& Rodgers M (2012). "A review of wearable sensors and systems with application in rehabilitation." Journal of Neuroengineering and Rehabilitation, 9, 21.

Pereira CLN, Vogelaere P, \& Baptista F (2008). "Role of physical activity in the prevention of falls and their consequences in the elderly." European Review of Aging and Physical Activity, 5(1), 51-58.

Pijnappels M, van der Burg PJCE, Reeves ND, \& van Dieën JH (2008). "Identification of elderly fallers by muscle strength measures.” European Journal of Applied Physiology, 102(5), 585-592.

Prochaska JO, \& Velicer WF (1997). The Transtheoretical model of health behavior change. American Journal of Health Promotion, 12(1), 38-48.

Psaltopoulou T, Kyrozis A, Stathopoulos P, Trichopoulos D, Vassilopoulos D, \& Trichopoulou A (2008). "Diet, physical activity and cognitive impairment among elders: The EPIC-Greece cohort (European prospective investigation into Cancer and nutrition)." Public Health Nutrition, 11(10), 1054-1062.

Satariano WA, Wang C, Kealey ME, Kurtovich E, \& Phelan EA (2017). "Risk profiles for falls among older adults: New directions for prevention.” Frontiers in Public Health, 5, 142. 
Sawchuk CN, Russo JE, Bogart A, Charles S, Goldberg J, Forquera R, Roy-Byrne P, \& Buchwald D (2011). "Barriers and facilitators to walking and physical activity among American Indian elders." Preventing chronic disease, 8(3), A63.

Sherrington C, Whitney JC, Lord SR, Herbert RD, Cumming RG, \& Close JCT (2008). "Effective exercise for the prevention of falls: A systematic review and meta-analysis." Journal of the American Geriatrics Society, 56(12), 2234-2243.

Shirly Ryan Ability Lab (2013). "30 Second Sit to Stand Test." Retrieved October 10, 2019 (https://www. sralab.org/rehabilitation-measures/30-second-sit-stand-test).

Shubert TE (2011). "Evidence-based exercise prescription for balance and falls prevention: A current review of the literature." Journal of Geriatric Physical Therapy, 34(3), 100-108.

Silva PA, Nunes F, Vasconcelos A, Kerwin M, Moutinho R, Teixeira P (2013). "Using the Smartphone Accelerometer to Monitor Fall Risk while Playing a Game: The Design and Usability Evaluation of Dance! Don't Fall.” In: Schmorrow D.D., Fidopiastis C.M. (eds) Foundations of Augmented Cognition. AC 2013. Lecture Notes in Computer Science, vol 8027, 754-763. Springer, Berlin, Heidelberg.

Sneha S, \& Varshney U (2009). "Enabling ubiquitous patient monitoring: Model, decision protocols, opportunities and challenges.” Decision Support Systems, 46(3), 606-619.

Taylor MJD, Shawis T, Impson R, Ewins K, McCormick D, \& Griffin M (2012). "Nintendo Wii as a training tool in falls prevention rehabilitation: Case studies." Journal of the American Geriatrics Society, 60(9), 1781-1783.

Tinetti ME, Speechley M, \& Ginter SF (1988). "Falls among elderly persons." The New England Journal of Medicine, 319(26), 1701-1706.

United Nations - Population Division Department of Economic and Social Affairs. (2017). World Population Ageing, 2017. https://www.un.org/en/development/desa/population/publications/pdf/ageing/WPA2017_ Report.pdf. Accessed 21 Sept 2019

Uzor S, Baillie L, Skelton DA, \& Rowe PJ (2013). "Falls prevention advice and visual feedback to those at risk of falling: Study protocol for a pilot randomized controlled trial." Trials, 14(1), 79.

Williams MA, Soiza RL, Jenkinson AME, \& Stewart A (2010). "EXercising with Computers in Later L ife (EXCELL) - pilot and feasibility study of the acceptability of the Nintendo® WiiFit in communitydwelling fallers." BMC Res Notes 3, 238.

World Health Organisation (2007). "WHO Global Report on Falls Prevention in Older Age." https://extranet. who.int/agefriendlyworld/wp-content/uploads/2014/06/WHo-Global-report-on-falls-prevention-in-olderage.pdf. Accessed 21 Sept 2019.

World Health Organisation (2018). "Falls." (https:/www.who.int/news-room/fact-sheets/detail/falls). Accessed 21 Sept 2019

Yu M, Rhuma A, Naqvi SM, Liang W, \& Chambers J (2012). “A posture recognition based fall detection system for monitoring an elderly person in a smart home environment." IEEE Transactions on Information Technology in Biomedicine : A Publication of the IEEE Engineering in Medicine and Biology Society, 16(6), 1274-1286.

Zijlstra G, van Haastregt J, van Eijk J, \& Kempen G (2005). "Evaluating an Intervention to Reduce Fear of Falling and Associated Activity Restriction in Elderly Persons: Design of a Randomised Controlled Trial [ISRCTN43792817].” BMC Public Health 5, Article number: 26.

Publisher's Note Springer Nature remains neutral with regard to jurisdictional claims in published maps and institutional affiliations. 